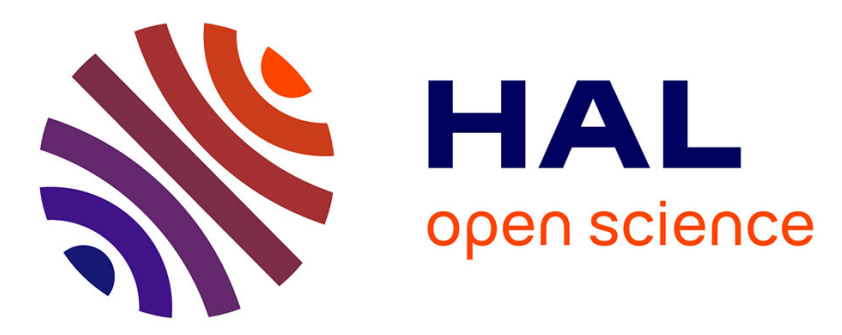

\title{
Notes on the Extraction of Aspect-Independent Parameters of Chipless RFID Tags
}

\author{
Zeshan Ali, Etienne Perret
}

\section{To cite this version:}

Zeshan Ali, Etienne Perret. Notes on the Extraction of Aspect-Independent Parameters of Chipless RFID Tags. 2021 6th International Conference on Smart and Sustainable Technologies (SpliTech), Sep 2021, Bol and Split, Croatia. 10.23919/SpliTech52315.2021.9566393 . hal-03448431

\section{HAL Id: hal-03448431 https://hal.science/hal-03448431}

Submitted on 25 Nov 2021

HAL is a multi-disciplinary open access archive for the deposit and dissemination of scientific research documents, whether they are published or not. The documents may come from teaching and research institutions in France or abroad, or from public or private research centers.
L'archive ouverte pluridisciplinaire HAL, est destinée au dépôt et à la diffusion de documents scientifiques de niveau recherche, publiés ou non, émanant des établissements d'enseignement et de recherche français ou étrangers, des laboratoires publics ou privés. 


\title{
Notes on the Extraction of Aspect-Independent Parameters of Chipless RFID Tags
}

\author{
Zeshan Ali $^{*}$ and Etienne Perret ${ }^{* \dagger}$ \\ ${ }^{*}$ Univ. Grenoble Alpes, Grenoble INP, LCIS, 26000 Valence, France \\ ${ }^{\dagger}$ Institut Universitaire de France, 75005 Paris, France \\ zeshan.ali@1cis.grenoble-inp.fr, etienne.perret@lcis.grenoble-inp.fr
}

\begin{abstract}
This paper presents the extraction of aspectindependent parameters for dual-L dipoles based chipless radio frequency (RF) identification (RFID) tags using spectrogram method using single measurement. The implemented spectrogram method is based on a single short-time Fourier transform (STFT) averaging window. The performance of the spectrogram method is compared to the matrix pencil method (MPM). A computational time comparison between the spectrogram method and MPM is presented.
\end{abstract}

Index Terms-Chipless radio frequency (RF) identification (RFID), matrix pencil method (MPM), quality factor, short time Fourier transform (STFT).

\section{INTRODUCTION}

The robust detection techniques are indispensable for the chipless radiofrequency (RF) identification (RFID) to make it as a potential tool for item level tagging [1]. Several approaches have been proposed in the literature aiming for the robust detection of chipless RFID tags. These approaches include matrix pencil method (MPM) [2], short time matrix pencil method (STMPM) [3], short time Prony analysis (STPA) [4], inverse synthetic aperture radar (ISAR) data processing [5], scalar method [6], short time Fourier transform (STFT) [7], and spectrogram method [8].

In [8], we have presented the extraction of aspectindependent parameters [analogous to complex natural resonances (CNRs)] for $45^{\circ}$ shorted dipoles based chipless RFID tags using single measurement in practical environment. These extractions have been performed at various aspect angles along with various objects.

In this paper, we are presenting the extraction of aspectindependent parameters for dual-L dipoles based chipless RFID tags using single measurement in anechoic environment. The implemented spectrogram method in this paper is slightly different from the one in [8], as this spectrogram method is based on a single STFT averaging window for all scatterers as compared to the spectrogram method in [8] based on the dedicated STFT averaging window for each scatterer. The performance of the spectrogram method is compared to MPM. A computation time comparison between the spectrogram method and MPM is also done.

The organization of this paper is as follows. Section II presents the experimental measurements. Section III presents the extraction of aspect-independent parameters. Section IV presents a comparison of computational time durations between MPM and spectrogram method. Section V draws conclusions.

\section{EXPERIMENTAL MEASUREMENTS OF DEPOLARIZING CHIPLESS RFID TAGS}

This paper is aiming to present the extraction of aspectindependent parameters of depolarizing chipless RFID tags. For this purpose, the depolarizing RF Elementary Particle (REP) based chipless RFID tags are taken from the literature [9]. These depolarizing chipless RFID tags are based on dual-L dipoles. The precise geometric dimensions of six dual-L dipoles tag are outlined in [9], [10]. The experimental measurements of the chosen chipless RFID tags are performed using a vector network analyzer (VNA) based chipless RFID reader. The photographs of measurement setup and the employed chipless RFID tags are presented in Fig. 1. The chipless RFID reader is in the form of monostatic radar configured using an Agilent N5222A VNA and a Satimo QH2000 dual polarized antenna. The output power of VNA is $-5 \mathrm{dBm}$. The ports 1 and 2 of VNA are connected to the vertical and horizontal polarizations of the Satimo QH2000 antenna. The Satimo QH2000 presents the gain around $12 \mathrm{dBi}$ for the frequency sweep from $3 \mathrm{GHz}$ to $8 \mathrm{GHz}$, where the isolation between the two ports is larger than $30 \mathrm{~dB}$. The base of Satimo QH2000 is attached to a digitally controlled servo motor for varying the reading interrogation angle $\phi_{\mathrm{T}}$. The chipless RFID tags are placed at antenna to tag distance equals $10 \mathrm{~cm}$. The transmission coefficient $S_{21}$ is measured for both chipless RFID tags.

Here, we have presented an example of the reading error using a single scatterer based tag: single dual-L dipole tag. Fig. 2 shows the measured uncalibrated signals $\left|S_{21}\right|$ [see Fig. 2(a)] and the measured calibrated signals [see Fig. 2(b)] for the single dual-L dipole tag in an anechoic environment at two reader interrogation signal angle $\phi_{\mathrm{T}}$ : $15^{\circ}$ and $-15^{\circ} . \phi_{\mathrm{T}}$ is varied from the reader antenna while keeping the chipless RFID tag fixed at its position. The uncalibrated signals are without background normalization (i.e., postprocessing is not applied). The calibrated signals are with background normalization, where the background clutter is removed by subtracting the empty-signal (taken in the absence of the tag) measured at $\phi_{\mathrm{T}}=0^{\circ}$ from the tag-signals (taken in the presence of the tag) measured at $\phi_{\mathrm{T}}=15^{\circ}$ and $\phi_{\mathrm{T}}=-15^{\circ}$. The empty-signal is purposely measured at $\phi_{\mathrm{T}}=0^{\circ}$ to produce a reading error. For this reason, it is a partial removal of the clutter. In Fig. 2(a), it can be observed from both of the uncalibrated signals (i.e., without background normalization), the peak apexes linked to 


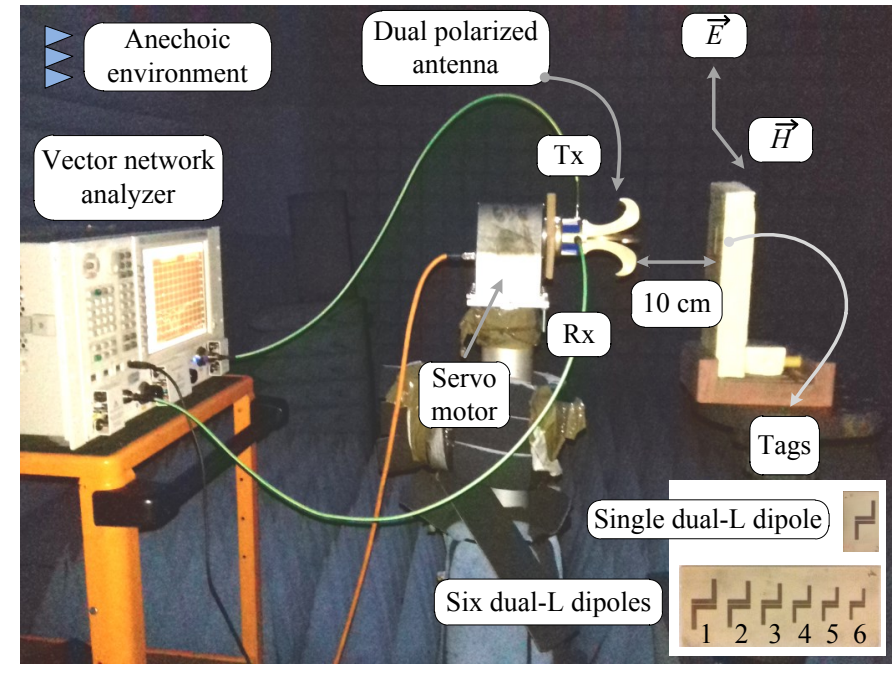

Fig. 1. Measurement setup for the experimental measurements of chipless RFID tags inside an anechoic environment. Inset: the photographs of the employed depolarizing chipless tags.

the frequency of resonance $f_{r}$ are not clearly visible due to the presence of the clutter (background information). If we consider the humps (that are followed by the dips) as peak apexes, the shift between them due to the change in $\phi_{\mathrm{T}}$ is around $15.5 \mathrm{MHz}$. The shift between the dips is $4 \mathrm{MHz}$. After removal of clutter in Fig. 2(b), the peak apexes that could be associated to the frequency of resonance $f_{r}$ are clearly visible in the calibrated signals (i.e., with background normalization) exhibiting a shift of 24.5 MHz. Even so, it can be observed that due to the change in $\phi_{\mathrm{T}}$ (i.e., reading error), the dips associated with the peak apexes exhibit a shift of $80.1 \mathrm{MHz}$ as $\phi_{\mathrm{T}}$ changes from $15^{\circ}$ to $-15^{\circ}$. These results show that the peak apexes of calibrated signals [Fig. 2(b)] are not precisely associated to $f_{r}$ of the scatterers, because these signals are still composed of the aspectdependent information. These reading challenges can be solved by extracting the aspect-independent parameters of chipless RFID tags by using appropriate postprocessing techniques. Indeed, if such an approach is applied to the uncalibrated signals of Fig. 2(a), a unique frequency which thus can be considered to be the scatterer's frequency of resonance $f_{r}$, equal to $4.16 \mathrm{GHz}$ (ideal peak apex's position) can be extracted.

In a tag reading process, the target tag is always known that can provide specific a priori information about the expected precise positions of the peak apexes corresponding to $f_{r i}$ of $i$ th scatterer. In Fig. 2, we have shown that the calibrated responses (background normalized) still contain the aspect-dependent information. Practically speaking, the optimum method to remove the aspect-dependent part of information and to extract the frequency of resonance $f_{r}$ and the quality factor $Q$ of the tag's resonant scatterers is:

- About the measurement, to use an anechoic chamber and a VNA (with the parameters given in Fig. 1).

- About the post-treatment, to do a background normalization (subtraction of the empty measurement from the tag measurement, where between these two measurements no

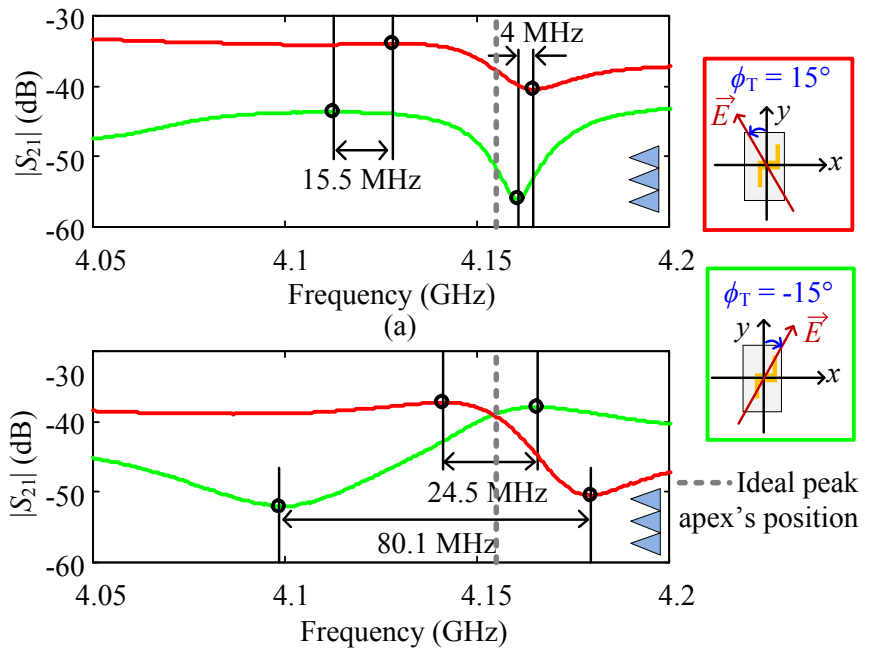

(b)

Fig. 2. Measured $\left|S_{21}\right|$ for the single dual-L dipole in an anechoic environment at two reader interrogation signal angles $\phi_{\mathrm{T}}: 15^{\circ}$ to $-15^{\circ}$. (a) The uncalibrated responses (without background normalization). (b) The calibrated responses (with background normalization).

TABLE I

Extracted A Priori Parameters.

\begin{tabular}{|c|c|c|c|c|c|c|c|}
\hline \multirow{2}{*}{ Tags } & \multirow{2}{*}{$\begin{array}{c}\text { a priori } \\
\text { parameters }\end{array}$} & \multicolumn{7}{|c|}{ Scatterers } \\
\cline { 2 - 8 } & & 1 & 2 & 3 & 4 & 5 & 6 \\
\hline Single & $f_{r i}^{\text {ap }}(\mathrm{GHz})$ & 4.16 & & & & & \\
dual-L & $Q_{i}^{\text {ap }}$ & 109.34 & & & & & \\
\cline { 2 - 8 } dipole & $\sigma_{i}^{\text {ap }}\left(\times 10^{8}\right)$ & 1.18 & & & & & \\
\hline Six & $f_{r i}^{\text {ap }}(\mathrm{GHz})$ & 4.33 & 4.70 & 5.22 & 5.77 & 6.34 & 6.88 \\
\cline { 2 - 9 } dual-L & $Q_{i}^{\text {ap }}$ & 98.39 & 99.65 & 104 & 95 & 82.67 & 88.52 \\
\cline { 2 - 9 } dipoles & $\sigma_{i}^{\text {ap }}\left(\times 10^{8}\right)$ & 1.38 & 1.48 & 1.58 & 1.91 & 2.41 & 2.44 \\
\hline
\end{tabular}

change in the reader configuration is done) and a time windowing to suppress part of the early time response where signal to noise ratio is very low (i.e., the proportion of the tag response compared to, for example, the antenna coupling is low).

The extracted a priori parameters associated with each scatterer of employed tags using the approach described above, are outlined in Table I, where the order of the scatterers is taken from largest scatterer to smallest scatterer [see Fig. 1(inset)]. The extracted information (see Table I) will be used as the reference in the entire paper.

\section{EXtraction of Aspect-IndePendent PaRAmeters}

In this Section, the uncalibrated signals (i.e., without background normalization) measured in an anechoic environment are used.

\section{A. Matrix Pencil Method}

The complete list of steps of the algorithm of MPM for calculating the complex poles and residues can be seen in [11]. Fig. 3 shows a flowchart of the extraction of physical poles by MPM. 


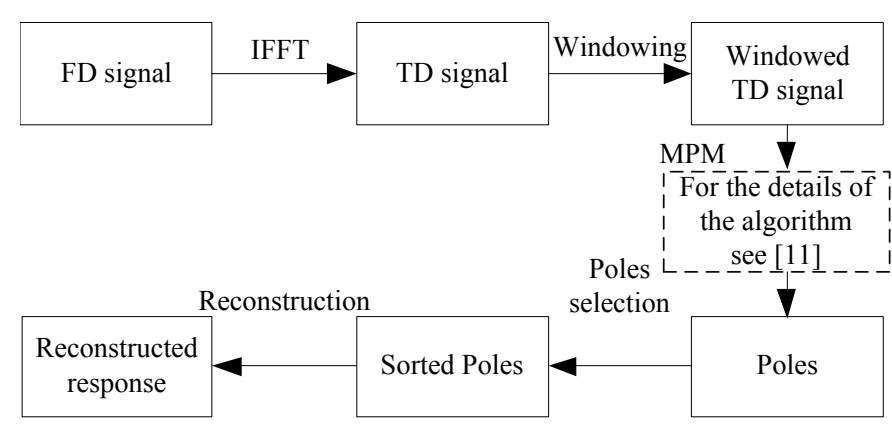

Fig. 3. Flowchart of the extraction of CNRs using matrix pencil method.

The uncalibrated signal is used to extract CNRs of the single dual-L dipole tag [see Fig. 1(inset)]. Fig. 4 shows the time windowing procedure to extract the sufficient late time response of the tag by applying a window of a time duration of $20 \mathrm{~ns}$, where the beginning of late time $T_{\mathrm{LB}}=2 \mathrm{~ns}$ and the ending of late time $T_{\mathrm{LE}}=22 \mathrm{~ns}$. The late time $T_{\mathrm{LE}}=22$ is estimated based on a priori extracted parameters (see Table I). This time windowing is carried by calculating inverse fast Fourier transform (IFFT) of measured uncalibrated frequency domain (FD) signal (non-windowed signals, solid green line), applying truncation in TD, and calculating fast Fourier transform (FFT) of truncated TD signal (windowed signal, dashed red line). The elimination of $2 \mathrm{~ns}$ (i.e., $T_{\mathrm{LB}}=2 \mathrm{~ns}$ ) of early part of TD signal is carried out to discard the direct reflections from the tag and its holder (the structural mode). Note that the early time elimination is mandatory to obtained accurate results with MPM. Windowed TD signal exhibiting 80 data points is used for the poles' extraction using MPM with $p=3$.

Then, further sorting of the poles is carried out by applying a filtering bandwidth of $100 \mathrm{MHz} B W_{100 \mathrm{MHz}}$ in a range of -50 $\mathrm{MHz} \leq f_{r i}^{\mathrm{ap}} \leq 50 \mathrm{MHz}$. Finally, a second-order bandpass filter model is used for the reconstruction of signals [8, eq. (12)].

Fig. 5(a) presents CNRs based MPM reconstructed FD response in comparison to the supplied windowed FD response and the non-windowed FD response. The extracted CNRs using MPM in comparison to a priori extracted parameters (like CNRs, see [8] for the demonstration) are shown in Fig. 5(b). The estimated CNRs from MPM are shown in the complex conjugate form. For the rest of this paper, only the positive CNRs would be shown for better visibility and better comparison.

\section{B. Spectrogram Method}

Fig. 6 shows a flowchart of the extraction of physical poles by spectrogram method. Fig. 7(a) shows the calculated STFT for single dual-L tag measured in an anechoic environment. The uncalibrated TD signal that is truncated at $100 \mathrm{~ns}$ [shown in Fig. 4(b), green curve] is supplied to the spectrogram method algorithm, where a hamming window of $30 \mathrm{~ns}$ with $90 \%$ overlap is used to compute STFT.

Fig. 7(a) shows also an area marked as black (within black dashed lines) for STFT averaging. To estimate this STFT averaging region, time width of STFT averaging window $T_{\text {avgW }}$

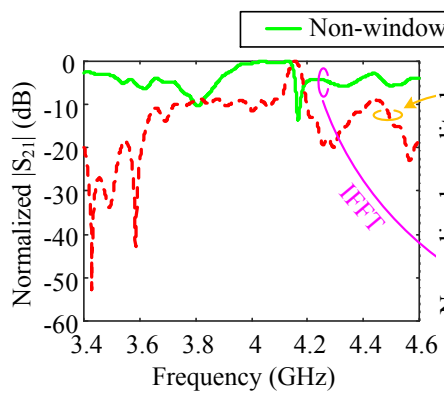

(a)

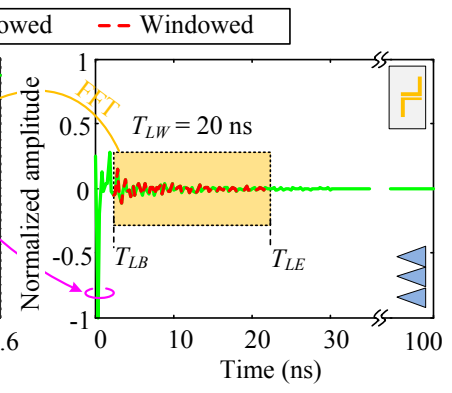

(b)
Fig. 4. Time windowing procedure for the single dual-L tag's uncalibrated response measured in an anechoic environment. (a) Frequency domain responses. (b) Time domain responses.

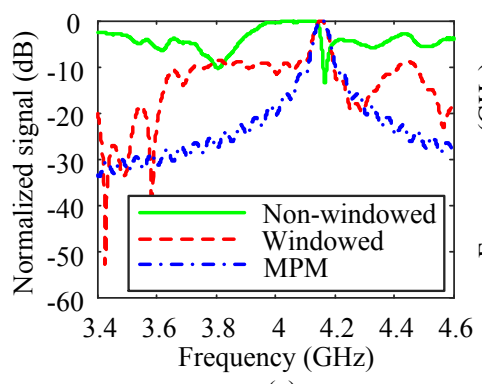

(a)

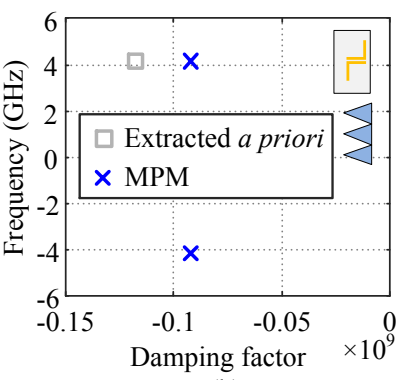

(b)
Fig. 5. Extraction of poles of single dual-L dipole tag using MPM measured in an anechoic environment with $p=3, T_{\mathrm{LW}}=20 \mathrm{~ns}$ and $B W_{100 \mathrm{MHz}}=$ $-50 \mathrm{MHz} \leq f_{r i}^{\text {ap }} \leq 50 \mathrm{MHz}$ and without the background normalization. (a) FD responses of reconstructed MPM response, non-windowed (uncalibrated) response and windowed (uncalibrated) response. (b) Extracted complex poles in comparison to extracted a priori complex poles (i.e., analogous to CNRs, see Table I).

can be calculated from the decaying envelope generated by using a priori extracted damping factors $\sigma_{i}^{\text {ap }}$ (see Table I). The time width of STFT averaging window $T_{\mathrm{avgW}}$ is based on the beginning time of STFT averaging window $T_{\mathrm{avgB}}=25 \mathrm{~ns}$ and the ending time of STFT averaging window $T_{\text {avgE }}=57.9 \mathrm{~ns}$, where $T_{\text {avgE }}$ is calculated using [8, eq. (10)] with a threshold amplitude $a_{e n}=0.001, T_{\text {avgE }}=t_{e n}^{\sigma_{1}^{\text {ap }}}$. Detailed discussions on the calculation $T_{\text {avgB }}$ and $T_{\text {avgE }}$ can be seen in [4], [8]. It is important to note that $T_{\text {avgB }}=25 \mathrm{~ns}$ in Fig. 7(a) (i.e., used to avoid the coupling in the averaging of STFT) is similar to $T_{\mathrm{LB}}=2 \mathrm{~ns}$ in Fig. 4 (i.e., used to discard the structural mode).

Then, the column vectors of STFT falling within $T_{\text {avgW }}$ (area marked as black) are averaged, as shown in Fig. 7(a). From the viewpoint of flow chart of the spectrogram method (Fig. 6), $f_{r i}$ related to $i$ th scatterer can be extracted from the peak apexes of STFT averaged ( $\mathrm{STFT}_{\text {avg }}$ ) signal. In the same way, Fig. 7(b) shows the extraction of $f_{r}$ from the peak apex of STFT $_{\text {avg }}$ signal for the single dual-L dipole tag.

Further, according to the flow chart of the proposed technique (Fig. 6), the damping factor $\sigma_{i}$ related to $i$ th scatterer can be calculated from the decaying time vector $A(\tau)_{i}$ residing inside $T_{\text {avgW }}$ at the extracted $f_{r i}$ (i.e., the position of the peak apex in $\mathrm{STFT}_{\text {avg }}$ signal). The damping factor $\sigma_{i}$ can be calculated by 


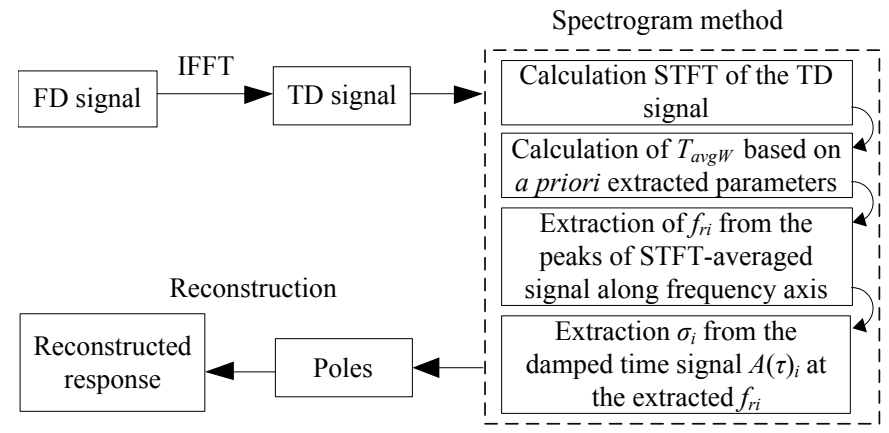

Fig. 6. Flowchart of the spectrogram method.

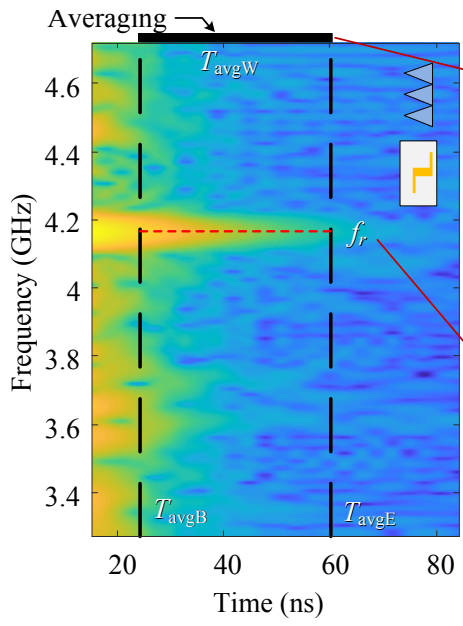

(a)

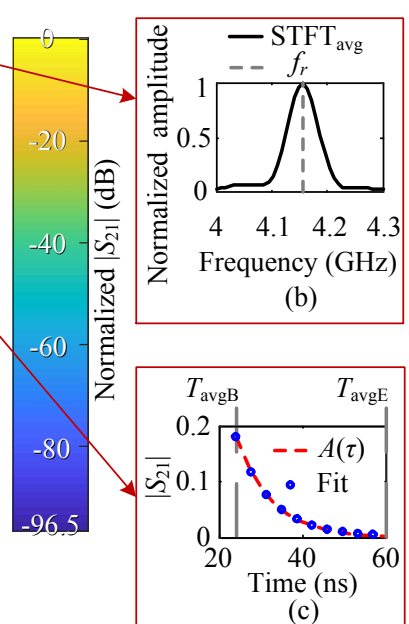

(c)
Fig. 7. Extraction of CNR by spectrogram method for single dual-L dipole tag using uncalibrated signal measured in an anechoic environment. (a) STFT calculated using a Hamming window of $30 \mathrm{~ns}$ with an overlap of $90 \%$. (b) Extraction of $f_{r}$ from the peak apex of STFT avg signal. (c) Extraction of $\sigma$ from the damping time signal $A(\tau)$ selected at $f_{r}$ inside $T_{\text {avgW }}$.

fitting or by least square method of solving the overdetermined linear system of equations as discussed in [8]. For the single dual-L dipole tag, the extraction of $\sigma$ from the damping time signal $A(\tau)$ selected at $f_{r}$ inside $T_{\text {avgw }}$ is presented in Fig. 7(c).

The difference between the extracted parameters using spectrogram approach (Fig. 7) and a priori extracted parameters (Table I) is that TD signal utilized for the extraction in spectrogram approach is uncalibrated (i.e., without background normalization) and without time windowing procedure. $f_{r i}$ and $\sigma_{i}$ parameters extracted by the spectrogram method are analogous to the poles (CNRs) extracted by using MPM.

Finally, a second-order bandpass filter model is used for the reconstruction of signals [8, eq. (12)].

Fig. 8(a) shows a comparison of reconstructed FD responses from both MPM and spectrogram method along with their corresponding supplied uncalibrated responses (i.e., windowed FD response and non-windowed FD response). The uncalibrated windowed TD response and uncalibrated non-windowed TD response are supplied to MPM and spectrogram method, respectively, where they are used to extract the aspect-independent

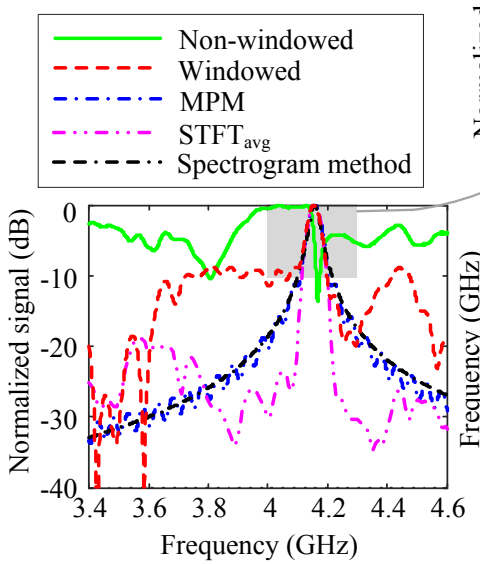

(a)

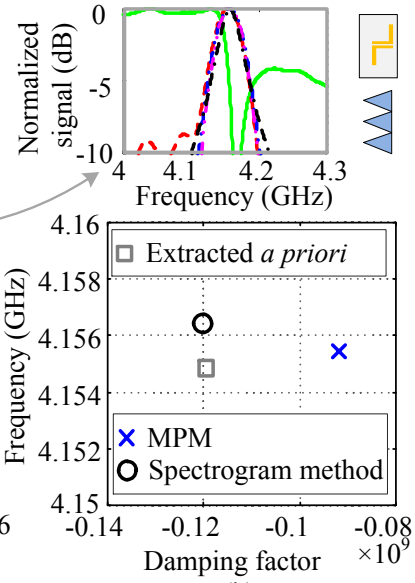

(b)
Fig. 8. Comparison of MPM and spectrogram method. (a) Reconstructed FD responses along with their corresponding supplied uncalibrated responses (i.e., windowed FD response and non-windowed FD response). The uncalibrated windowed TD response and uncalibrated non-windowed TD response are supplied to MPM and spectrogram method, respectively, where they used to extract the aspect-independent parameters for single dual-L dipole tag measured in an anechoic environment. (b) Extracted complex poles in comparison with a priori extracted complex pole.

parameters for single dual-L dipole tag measured in an anechoic environment. A zoom of Fig. 8(a) is provided to show the matching between reconstructed responses from both employed methods. Fig. 8(b) shows a comparison of extracted complex poles and a priori extracted complex pole. The extraction error of MPM extracted parameters with respect to a priori extracted parameters are $\left|\Delta f_{r}^{\mathrm{MP}}\right|=0.57 \mathrm{MHz},\left|\Delta \sigma^{\mathrm{MP}}\right|=0.02 \times 10^{9}$, and $\left|\Delta Q^{\mathrm{MP}}\right|=32.68$. The extraction error of spectrogram extracted parameters with respect to a priori extracted parameters are $\left|\Delta f_{r}^{\mathrm{SM}}\right|=1.54 \mathrm{MHz},\left|\Delta \sigma^{\mathrm{SM}}\right|=0.0006 \times 10^{9}$, and $\left|\Delta Q^{\mathrm{SM}}\right|=0.48$.

Note that the procedure to implement MPM is not straightforward because the choice of $T_{\mathrm{LB}}, T_{\mathrm{LE}}$, and $p$ must be proper. If care is not taken, numerous spurious poles can emerge, even after applying a filtering bandwidth of $100 \mathrm{MHz} B W_{100 \mathrm{MHz}}$ in a range of $-50 \mathrm{MHz} \leq f_{r i}^{\mathrm{ap}} \leq 50 \mathrm{MHz}$.

It is important to note that the spectrogram method does not entirely dependent on a priori extracted parameters for the calculation of STFT averaging window parameters. These parameters can also be decided without the information of $a$ priori parameters. We have tested that even if STFT averaging window parameters are taken as $T_{\mathrm{avgB}}=20 \mathrm{~ns}$ and $T_{\mathrm{avgE}}=$ $50 \mathrm{~ns}$, we found the extracted $f_{r i}$ and $\sigma_{i}$ parameters exactly same as presented in Fig. 8 for spectrogram method.

\section{Extraction of CNRs of the Multi-Scatterers Based Tags}

For extraction of aspect-independent parameters for the multi-scatterers based tags using the spectrogram method, the algorithm follows the same steps as explained in Fig. 6. It is important to note that here, like in the previous Section, the measured responses of six dual-L dipoles tag supplied to the spectrogram method are uncalibrated TD signals. 
Fig. 9 shows the extraction of CNRs by spectrogram method for six dual-L dipoles tag measured in an anechoic environment. The calculated STFT of the uncalibrated TD signal is shown in Fig. 9(a), where a hamming window of 30 ns with $90 \%$ overlap is used to compute STFT. In this multi-scatterer tag detection, $T_{\text {avgW }}$ is calculated by using a priori extracted parameters of the largest scatterer $n=6$ (i.e., operating at highest $f_{r}$ in the design of chipless RFID tag) and the smallest scatterer $n=1$ (i.e., operating at lowest $f_{r}$ in the design of chipless RFID tag). Using [8, eq. (10)] with a threshold amplitude $a_{e n}=0.001$, the parameters of STFT averaging window are calculated as $T_{\text {avgE }}=t_{e n}^{\sigma_{1}^{\text {ap }}}$ that is $49.93 \mathrm{~ns}$ and $T_{\text {avgB }}=t_{e n}^{\sigma_{6}^{\text {ap }}}-$ $6 \mathrm{~ns}$ that is $22.30 \mathrm{~ns}$. The reason behind the subtraction of $6 \mathrm{~ns}$ from $t_{e n}^{\sigma_{6}^{\mathrm{ap}}}$ in the calculation of $T_{\text {avgB }}$ is to provide a sufficient time to extract the damping factor, because $t_{e n}^{\sigma_{\mathrm{b}}^{\mathrm{ap}}}$ is the time at which the envelope reaches a threshold amplitude $a_{e n}=0.001$. The above-discussed method is one way to calculate the optimized parameters of STFT averaging window. Otherwise, these parameters can also be fixed to certain values.

Fig. 9(b) shows the extraction of $f_{r i}$ from the peak apexes of STFT avg signal. The extraction of $\sigma_{i}$ is carried out from the damping time $A(\tau)_{i}$ signal selected at each $f_{r i}$ within $T_{\text {avgW }}$. Extraction of $\sigma_{6}$ and $\sigma_{1}$, for example, is presented in Fig. 9(c) and Fig. 9(d), respectively.

For the extraction of aspect-independent parameters for the multi-scatterers based tags using MPM, the algorithm follows the same steps as explained in Fig. 3. Time windowing is applied to the uncalibrated TD signal measured in an anechoic environment with $T_{\mathrm{LW}}=16 \mathrm{~ns}$, where early part up to $T_{\mathrm{LB}}=2 \mathrm{~ns}$ is discarded similar to as explained in Fig. 4. The windowed TD signal exhibiting 160 data points is supplied to MPM with $p=3$ to extract CNRs. Then, further sorting of the poles is carried out applying a filtering bandwidth of $100 \mathrm{MHz}$ $B W_{100 \mathrm{MHz}}$ in a range of $-50 \mathrm{MHz} \leq f_{r i}^{\text {ap }} \leq 50 \mathrm{MHz}$.

Fig. 10(a) shows a comparison of reconstructed FD responses from both MPM and spectrogram method along with their corresponding supplied uncalibrated responses (i.e., windowed FD response and non-windowed FD response). The uncalibrated windowed TD response and uncalibrated nonwindowed TD response are supplied to MPM and spectrogram method, respectively, where they are used to extract the aspectindependent parameters for six dual-L dipoles tag measured in an anechoic environment. Fig. 10(b) shows a comparison of extracted complex poles and a priori extracted complex poles. The extraction errors of extracted parameters using both the spectrogram method and MPM from their corresponding $a$ priori extracted parameters are outlined in Table II.

\section{Comparison of Computational Time Durations Between Matrix Pencil Method and Spectrogram METHOD}

A comparison of computational time durations has been done between the spectrogram method and MPM. Single dualL dipole tag (see inset of Fig. 1) is used for the experimental results. The measurements are performed in an anechoic environment in a monostatic cross-polarization configuration

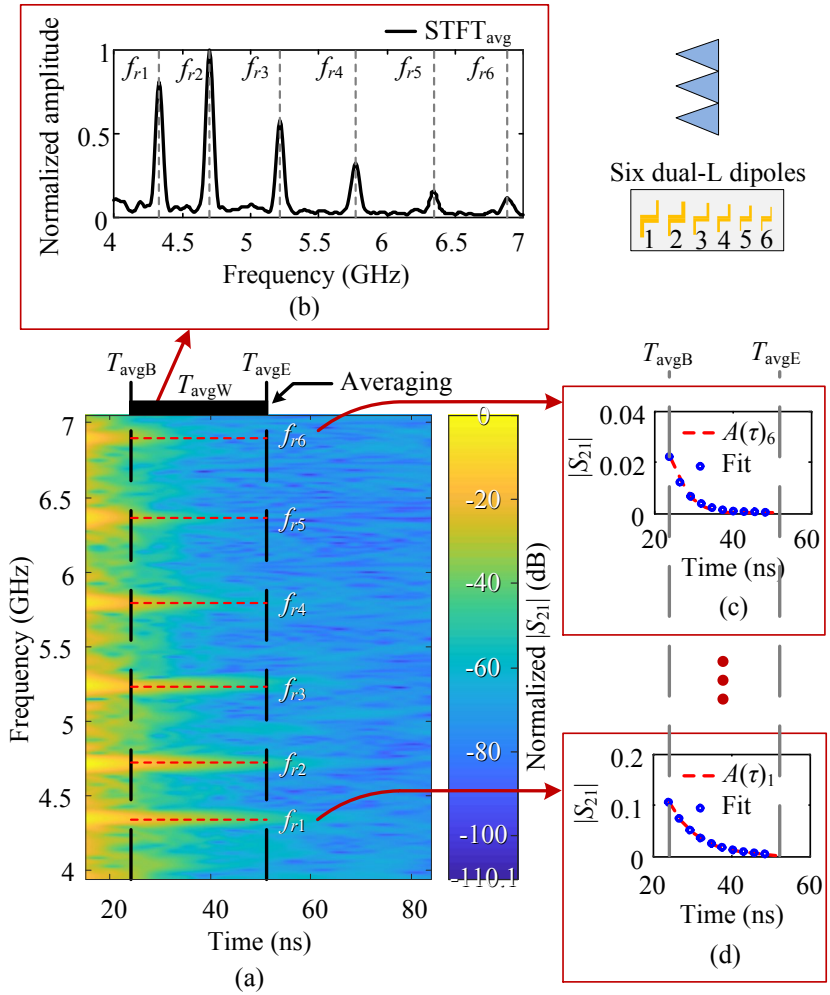

Fig. 9. Extraction of CNRs by spectrogram method for six dual-L dipoles tag using uncalibrated signal measured in an anechoic environment. (a) STFT calculated using a Hamming window of 30 ns with an overlap of $90 \%$. (b) Extraction of $f_{r i}$ from the peak apexes of STFT avg signal. (c) and (d) Extraction of $\sigma_{6}$ and $\sigma_{1}$ from the damping time signals $A(\tau)_{6}$ and $A(\tau)_{1}$ selected at $f_{r 6}$ and $f_{r 1}$ inside $T_{\text {avgW }}$, respectively.

TABLE II

EXTRACTION ERRORS OF SPECTROGRAM METHOD AND MPM IN AN ANECHOIC ENVIRONMENT.

\begin{tabular}{|c|c|c|c|c|c|c|}
\hline \multirow{2}{*}{ Scatterer $\boldsymbol{i}$} & \multicolumn{3}{|c|}{ Spectrogram method } & \multicolumn{3}{c|}{ Matrix Pencil Method } \\
\cline { 2 - 7 } & $\begin{array}{c}\left|\Delta f_{r i}\right| \\
(\mathrm{MHz})\end{array}$ & $\begin{array}{c}\left|\Delta \sigma_{i}\right| \\
\times 10^{9}\end{array}$ & $\left|\Delta Q_{i}\right|$ & $\begin{array}{c}\left|\Delta f_{r i}\right| \\
(\mathrm{MHz})\end{array}$ & $\begin{array}{c}\left|\Delta \sigma_{i}\right| \\
\times 10^{9}\end{array}$ & $\left|\Delta Q_{i}\right|$ \\
\hline 1 & $\approx 0$ & 0.002 & 1.48 & 3.90 & 0.01 & 8.13 \\
\hline 2 & $\approx 0$ & $\approx 0$ & 0.23 & 4.27 & 0.028 & 16.25 \\
\hline 3 & $\approx 0$ & 0.005 & 2.85 & 9.6 & 0.042 & 21.92 \\
\hline 4 & $\approx 0$ & 0.002 & 0.98 & 3.78 & 0.093 & 31.04 \\
\hline 5 & 9.76 & 0.013 & 4.36 & 3.67 & 0.022 & 8.37 \\
\hline 6 & $\approx 0$ & 0.02 & 8.29 & 2.74 & 0.103 & 26.3 \\
\hline
\end{tabular}

as shown in Fig. 1. Fig. 11 shows TD responses for the single dual-L tag calculated from the calibrated FD response measured in an anechoic environment. To calculate the computational time durations, time windowing is applied to take the signal in various lengths. The beginning time of time window $T_{\mathrm{LB}}$ is kept fixed to discard the structural mode, while the ending time of time windows $T_{\mathrm{LE}}$ is varied to take different lengths of the signal. We have taken the window length from $100 \mathrm{~ns}$ to $1000 \mathrm{~ns}$ with equal steps of $100 \mathrm{~ns}$. To calculate the computational time durations, both methods (spectrogram method and MPM) are fed by the same windowed signal (i.e., exhibiting equal length in time duration). The calculated computational time 


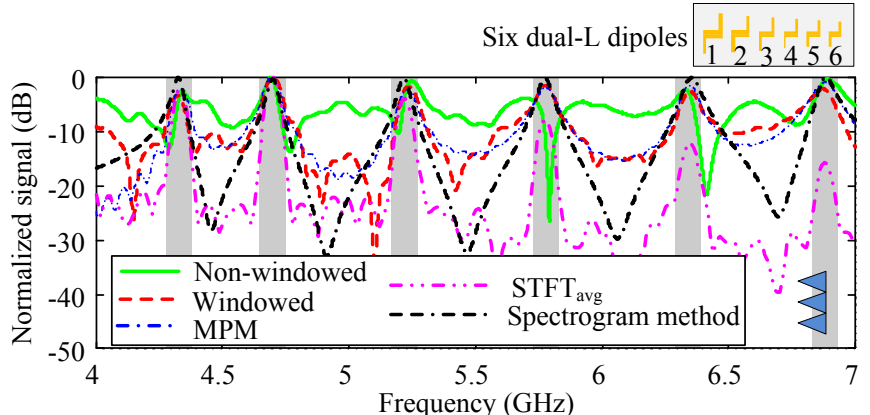

(a)

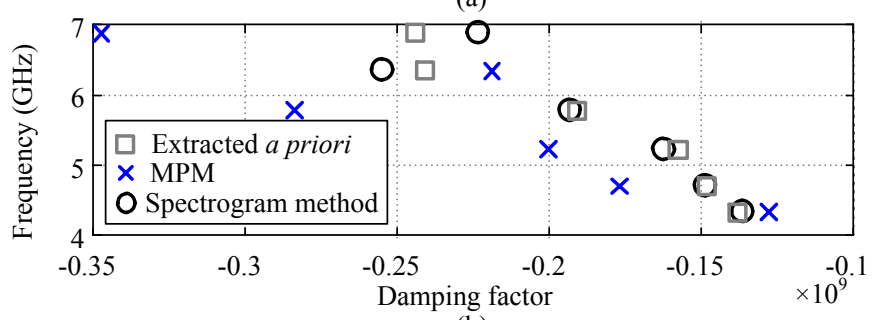

(b)

Fig. 10. Comparison of MPM and spectrogram method. (a) Reconstructed FD responses along with their corresponding supplied uncalibrated responses (i.e. windowed FD response and non-windowed FD response). The uncalibrated windowed TD response and uncalibrated non-windowed TD response are supplied to MPM and spectrogram method, respectively, where they are used to extract the aspect-independent parameters for six dual-L dipoles tag measured in an anechoic environment. (b) Extracted complex poles in comparison with a priori extracted complex poles. Shaded areas are in the range of $-50 \mathrm{MHz} \leq f_{r i}^{\mathrm{ap}} \leq 50 \mathrm{MHz}$.

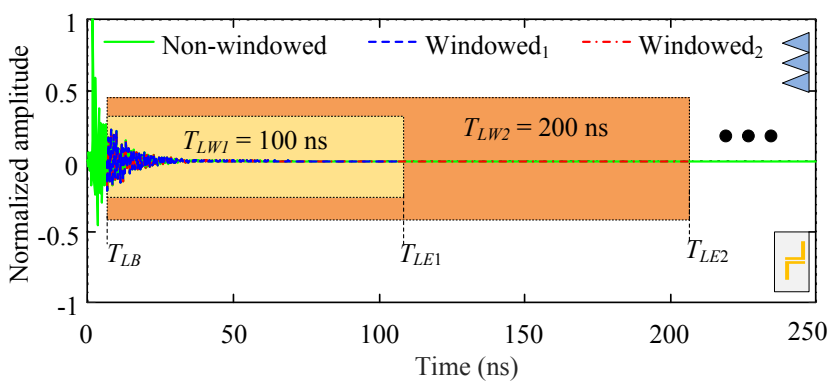

Fig. 11. Windowed TD responses with window length $T_{\mathrm{LW}}$ ranging from $100 \mathrm{~ns}$ to $1000 \mathrm{~ns}$ with a step size of $100 \mathrm{~ns}$ for the single dual-L dipole tag calculated from the calibrated FD response measured in an anechoic environment.

durations for both methods (spectrogram method and MPM) using different windows' lengths are presented in Fig. 12. This calculation is conducted using MATLAB on an Intel (i7$5600 \mathrm{U})$ processor. We found that MPM is computationally very expensive as compared to the spectrogram method. For a window of $100 \mathrm{~ns}$, the spectrogram method took around $4.7 \mathrm{~ms}$, while MPM took $28.5 \mathrm{~ms}$. For a window of $1000 \mathrm{~ns}$, the spectrogram method took $37.8 \mathrm{~ms}$, while MPM method took $9.15 \mathrm{~s}$.

\section{CONClusion}

The extraction of aspect-independent parameters for dual-L dipoles based chipless RFID tags using single measurement

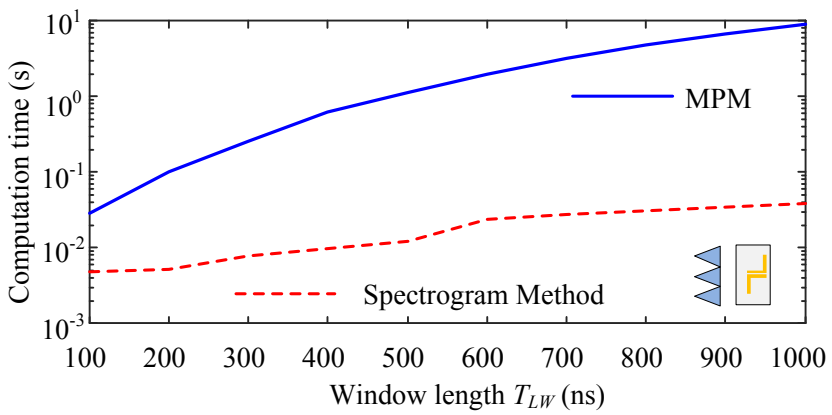

Fig. 12. Comparison of computational time durations between the spectrogram method and MPM.

in anechoic environment was presented in this paper. The performance of the spectrogram method is compared to the matrix pencil method (MPM). A computation time comparison between the spectrogram method and MPM was done. The spectrogram method was computationally fast as compared to MPM due to fundamental characteristic of FFT.

\section{ACKNOWLEDGMENT}

This work is funded by the European Research Council (ERC) under the European Union's Horizon 2020 research and innovation program under grant agreement no. 772539 (SCATTERERID, https://www.scattererid.eu/).

\section{REFERENCES}

[1] E. Perret, Radio Frequency Identification and Sensors: From RFID to Chipless RFID. Hoboken, NJ, USA: Wiley: Wiley, 2014.

[2] A. T. Blischak and M. Manteghi, "Embedded Singularity Chipless RFID Tags," IEEE Trans. Antennas Propag., vol. 59, no. 11, pp. 3961-3968, Nov. 2011.

[3] R. Rezaiesarlak and M. Manteghi, "Accurate Extraction of Early-/LateTime Responses Using Short-Time Matrix Pencil Method for Transient Analysis of Scatterers," IEEE Trans. Antennas Propag., vol. 63, no. 11, pp. 4995-5002, Nov. 2015.

[4] R. Tavares de Alencar, Z. Ali, N. Barbot, M. Garbati, and E. Perret, "Practical Performance Comparison of 1-D and 2-D Decoding Methods for a Chipless RFID System in a Real Environment," IEEE J. Radio Freq. Identification, vol. 4, no. 4, pp. 532-544, Dec. 2020.

[5] F. Costa, M. Borgese, A. Gentile, L. Buoncristiani, S. Genovesi, F. A. Dicandia, D. Bianchi, A. Monorchio, and G. Manara, "Robust Reading Approach for Moving Chipless RFID Tags by Using ISAR Processing," IEEE Trans. Microw. Theory Techn., vol. 66, no. 5, pp. 2442-2451, May 2018.

[6] J. Kracek, M. Svanda, and K. Hoffmann, "Scalar Method for Reading of Chipless RFID Tags Based on Limited Ground Plane Backed Dipole Resonator Array," IEEE Trans. Microw. Theory Techn., vol. 67, no. 11, pp. 4547-4558, Nov. 2019.

[7] A. Ramos, Z. Ali, A. Vena, M. Garbati, and E. Perret, "Single-layer, Flexible, and Depolarizing Chipless RFID Tags," IEEE Access, 2020.

[8] Z. Ali, E. Perret, N. Barbot, and R. Siragusa, "Extraction of AspectIndependent Parameters Using Spectrogram Method for Chipless Frequency-Coded RFID," IEEE Sensors J., vol. 21, no. 5, pp. 65306542, 2021.

[9] A. Vena, E. Perret, and S. Tedjni, "A Depolarizing Chipless RFID Tag for Robust Detection and Its FCC Compliant UWB Reading System," IEEE Trans. Microw. Theory Techn., vol. 61, no. 8, pp. 2982-2994, Aug. 2013.

[10] Z. Ali and E. Perret, "A Simple RCS Calibration Approach For Depolarizing Chipless RFID Tags," in Proc. IEEE MTT-S Int. Microw. Symp. Dig., Atlanta, GA, USA, Jun. 2021.

[11] T. K. Sarkar and O. Pereira, "Using the matrix pencil method to estimate the parameters of a sum of complex exponentials," IEEE Antennas Propag. Mag., vol. 37, no. 1, pp. 48-55, Feb. 1995. 